\title{
Joint Scalable Video Coding and Packet Prioritization for Video Streaming over IP/802.11e Heterogeneous Networks
}

\author{
Thomas Pliakas \\ Dept. of Information \& \\ Communication Systems \\ Engineering \\ University of Aegean \\ Karlovasi, Samos, Greece \\ tpliakas@aegean.gr
}

\author{
George Kormentzas \\ Dept. of Information \& \\ Communication Systems \\ Engineering \\ University of Aegean \\ Karlovasi, Samos, Greece \\ gkorm@aegean.gr
}

\author{
Sofia Tsekeridou \\ Athens Information \\ Technology - AIT \\ Building B7, $19.5 \mathrm{~km}$ \\ Markopoulo Ave \\ Peania, Athens, Greece \\ sots@ait.edu.gr
}

\begin{abstract}
The paper discusses scalable video streaming traffic delivery over heterogeneous DiffServ/WLAN networks. A prototype architecture is proposed and further validated that explores the joint use of packet prioritization and scalable video coding (SVC) together with the appropriate mapping of 802.11e access categories to the DiffServ traffic classes. A complete set of simulation scenarios, involving four different video sequences using the scalable extension of H.264/MPEG-4 AVC, demonstrates the quality gains of both scalable video codingand prioritized packetization.
\end{abstract}

\section{Keywords}

802.11e, DiffServ, End-to-End QoS, H.264/MPEG-4 AVC, Packet Prioritization, SVC, WLAN

\section{INTRODUCTION}

IP technology seems to be able to resolve the inter-working amongst the diverse fixed core and wireless access technologies. At the network level, the end-to-end QoS provision could be established through the appropriate mapping amongst the QoS traffic classes/services supported by the contributing underlying networking technologies [11] [12]. A QoS cross layer architecture based on error resilience features of H.264/MPEG-4 AVC can be applied for further improvements on end-to-end QoS. Building on this background, this work involves a DiffServ-aware IP core network and a 802.11e access network and examines end-toend QoS issues regarding scalable video streaming and prioritized packetization based on data partitioning (DP) for delivering multimedia traffic across fixed and wireless network domains.

The Differentiated Services (DiffServ) [4] approach proposed by IETF supports (based on the DiffServ Code Point (DSCP) [8] field of the IP header) two different services, the Expedited Forwarding (EF) that offers low packet loss and

Permission to make digital or hard copies of all or part of this work for personal or classroom use is granted without fee provided that copies are not made or distributed for profit or commercial advantage and that copies bear this notice and the full citation on the first page. To copy otherwise, to republish, to post on servers or to redistribute to lists, requires prior specific permission and/or a fee. MOBIMEDIA 2007, August 27-29, Nafpaktos, Greece

Copyright (C) 2007 ICST 978-963-06-2670-5

DOI 10.4108/ICST.MOBIMEDIA2007.1854 low delay/jitter and the Assured Forwarding (AF), which provides better QoS guarantees than the best-effort service. Differences amongst AF services imply that a higher QoS AF class will give a better performance (faster delivery, lower loss probability) than a lower AF class.

The 802.11e [3] standard addresses the issue of QoS support in wireless LANs. The MAC protocol of 802.11e supports multiple access categories (ACs). A higher priority access category has a smaller minimum contention window thus has a higher probability to access the channel. Different access categories can have a different maximum contention window and inter-frame spacing interval (IFS). The 802.11e defines four access categories; AC3 corresponds to the highest access priority, and $\mathrm{AC} 0$ to the lowest.

The basic coding scheme for achieving a wide range of spatio-temporal and quality scalability is scalable video. For Signal-to-Noise Ratio (SNR) scalability the most appropriate technique for video delivery over heterogeneous networks, is the scalable extension of H.264/MPEG-4 AVC [13]. In order to support fine-granular SNR scalability, progressive refinement (PR) slices have been introduced in the scalable extension of H.264 [14]. A base representation of the input frames of each layer is obtained by transform coding similar to H.264 [18]. The corresponding Network Abstraction Layer (NAL) units (containing motion information and texture data) of the base layer are compatible with the single layer H.264/MPEG-4 AVC. Furthermore, by employing data partitioning, the H.264 encoder partitions the compressed data in separate units of different importance. The packets, with assigned priority, are sent to a QoS-aware network to receive different forwarding treatments. Mapping these prioritized packets to different QoS levels causes them to experience different packet loss rates with this differential forwarding mechanism. The quality of the base representation can be improved by an additional coding of the so-called PR slices. The corresponding NAL units can be arbitrarily truncated in order to support fine granular quality scalability or flexible bit-rate adaptation.

To address end-to-end QoS problem scalable video streaming traffic delivery over a heterogeneous IP/802.11e network, the paper proposes and validates through a number of NS2based simulation scenarios an architecture that explores the joint use of packet prioritization and scalable video coding together with the appropriate mapping of 802.11 e access categories to the DiffServ traffic classes. This work extends previous authors' papers [11] [12] dealing with joint scalable 


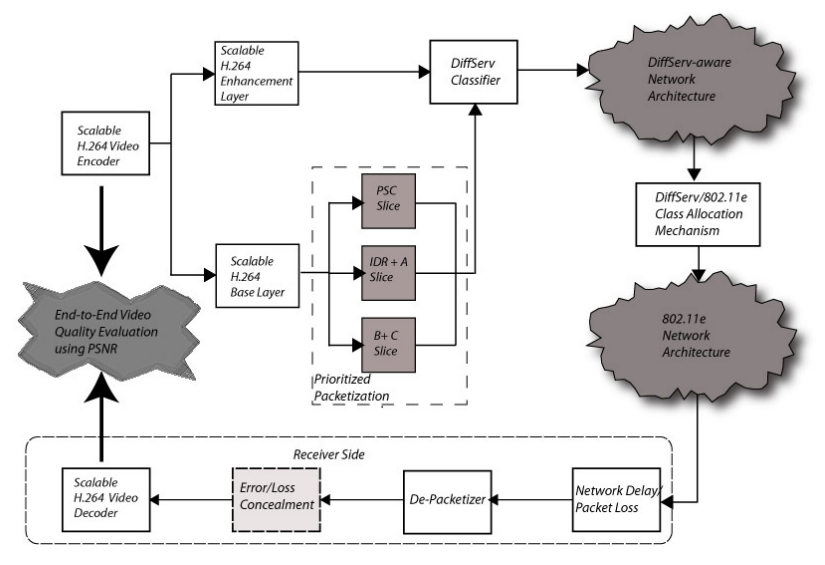

Figure 1: Overall Architecture

video coding and packet prioritization over IP/UMTS and IP/DVB heterogeneous networks.

The rest of the paper is organized as follows. In Section 2, the proposed scalable video coding techniques and prioritization framework for providing QoS guarantees for scalable video streaming traffic delivery over a heterogeneous DiffServ/WLAN network is presented. In Section 3, we demonstrate how video-streaming applications can benefit from the use of the proposed architecture. Finally, Section IV draws the conclusions and discusses directions for further work and improvements.

\section{PROPOSED ARCHITECTURE}

The proposed architecture integrates the concepts of scalable video streaming, prioritized packetization based on the H.264 data partitioning features and mapping DiffServ classes to MAC differentiation of 802.11e. The proposed architecture is depicted in Figure 1. It consists of three key components: (1) Scalable video encoding (Scalable extension of H.264/MPEG-4 AVC), (2) prioritized packetization according based on data partitioning, and (3) DiffServ/802.11e class mapping mechanism in order to assure the optimal differentiation and to achieve QoS continuity of scalable video streaming traffic delivery over DiffServ and 802.11e network domains. Each one of these components is discussed in detail in the following subsections.

\subsection{Scalable Video Coding}

Scalable Video Coding should meet a number of requirements in order to be suitable for multimedia streaming applications. For efficient utilization of available bandwidth, the compression performance must be high. Also, the computational complexity of the codec must be kept low to allow cost efficient and real time implementations. When compared against other scalable video coding schemes, the fine granular scalability coding method is outstanding due to its ability to adapt to changing network conditions more accurately.

\subsubsection{Scalable Extension of H.264/MPEG-4 AVC}

In order to provide FGS scalability, a picture must be represented by an H.264/AVC compatible base representation layer and one or more FGS enhancement representations, which demonstrate the residual between the original predic- tions residuals and intra blocks and their reconstructed base representation layer. This basic representation layer corresponds to a minimally acceptable decoded quality, which can be improved in a fine granular way by truncating the enhancement representation NAL units at any arbitrary point. Each enhancement representation contains a refinement signal that corresponds to a bisection of the quantization step size, and is directly coded in the transform coefficient domain.

For the encoding of the enhancement representation layers a new slice called Progressive Refinement (PR) has been introduced. In order to provide quality enhancement layer NAL units that can be truncated at any arbitrary point, the coding order of transform coefficient levels has been modified for the progressive refinement slices. The transform coefficient blocks are scanned in several paths, and in each path only a few coding symbols for a transform coefficient block are coded [16].

\subsection{Prioritized Packetization}

We define two groups of priority policies, one for BL and one for EL. These policies are used from the Edge Router of the DiffServ-aware underlying network to map the packets to the appropriate traffic classes. The packetization process can affect the efficiency as well as the error resiliency of video streaming.

For the BL, at the Video Coding Layer (VCL), an additional type of slice, besides the three partitions (A, B, and C) obtained when DP is enabled, that represents Instantaneous Decoding Refresh (IDR) pictures. The IDR access units contain information that cannot be included into the three partitions, like the intra-picture (coded picture that can be decoded without needing information from previous pictures) where no data partitioning can be applied.

The order in which the slice units are sent is constant. The first transmitted slice units transmitted contain the Packet Set Concept (PSC) information, such as picture size, display window, optional coding modes employed, macroblock allocation map, etc. This higher-layer meta information should be sent reliably, asynchronously, and before transmitting video slices.

The next transmitted slice units contain the IDR picture. Since IDR frames may contain only I slices without data partitioning, they are usually sent at the start of video sequences (just after the PSC). The slice units following the IDR frames contain one of the three partitions (A, B, or C).

The NAL is responsible for the encapsulation of the coded slices into transport entities of the network. Each NAL unit (NALU) could be considered as a packet that contains an integer number of bytes, including a header and a payload. The header specifies the NALU type, and the payload contains the related data. The most important field of the NAL header is the $N_{a l} R_{\text {Ref_Id }} I d$ (NRI) field [7].

The NRI contains two bits that indicate the priority of the NALU payload, where 11 is the highest transport priority, followed by 10 , then by 01 , and finally, 00 is the lowest. Accordingly, the incoming VCL layer slices are differentiated and encapsulated into NALUs by enabling the NRI field in the NAL header. Table 1 depicts the relation between the type of the BL content and the corresponding DiffServ classes. The first digit of the AF class indicates forwarding priority and the second indicates the packet drop precedence. 
Table 1: DiffServ Classes allocation for NRI

\begin{tabular}{|c|c|l|}
\hline DiffServ Classes & Slice Type & NRI Value \\
\hline EF & PSC & 11 \\
\hline AF11 & IDR A & 10 \\
\hline AF12 & B C & 01,00 \\
\hline
\end{tabular}

Table 2: DiffServ/802.11e classes coupling

\begin{tabular}{|c|c|l|}
\hline Traffic Class & DiffServ Classes & AC \\
\hline Class 1 & EF & 3 \\
\hline Class 2 & AF 11 & 2 \\
\hline Class 3 & AF 12 & 1 \\
\hline Class 4 & Best Effort & 0 \\
\hline
\end{tabular}

The PSC packets obtain the highest priority. Furthermore, as information carried in both partition A and IDR are essential for decoding an entire video frame, it is important to give these slices more priority than partition $\mathrm{B}$ and C. Based on these rules, the NAL layer marks the different NALUs.

\subsection{DiffServ/802.11e QoS Classes Coupling}

In order to integrate the 802.11 network domain with the core network domain, and to achieve QoS consistency across the DiffServ IP and 802.11e network, we have to map 802.11e access categories to predefined DiffServ classes. A direct mapping apprach as proposed by [10] is adopted. Table 2 shows the mapping of the predefined DiffServ classes according to the DiffServ specification, where the first digit of the AF class indicates forwarding priority and the second indicates the packet drop precedence, and the 802.11e access categories for the proposed mapping approach.

The packets, with assigned priority, are sent to the DiffServ network to receive different forwarding treatments. Mapping these prioritized packets to different QoS DS levels causes them to experience different packet loss rates with this differential forwarding mechanism. In addition to the prioritized dropping performed by DiffServ routers, traffic policing can be carried out at intermediate video gateways (between different network domains), using packet filtering. When the IP packets are encapsulated in MAC frames, each frame should be allocated to a priority queue, or an access category.

\section{FRAMEWORK EVALUATION}

This section evaluates the performance of the proposed framework through a set of simulations. A NS-2 based simulation environment with the appropriate extensions [1] for simulating $802.11 \mathrm{e}$ WLANs is adopted.

Four YUV QCIF 4:2:0 color video sequences consisting of 300 to 2000 frames and coded at 30 frames per second are used as video sources. Each group of pictures (GOP) is structured as IBBPBBPBB. and contains 36 frames, and the maximum UDP packet size is at 1024 bytes (payload only). The scalable extension of H.264/MPEG-4 AVC encoder/decoder provided by [2] is used for encoding YUV sequences. The video frames are then encapsulated into RTP packets using a simple packetization scheme [15] (by oneframe-one-packet policy). The size of each RTP packet is maximally bounded to 1024 bytes. The generated video packets are delivered through the DiffServ at the form of

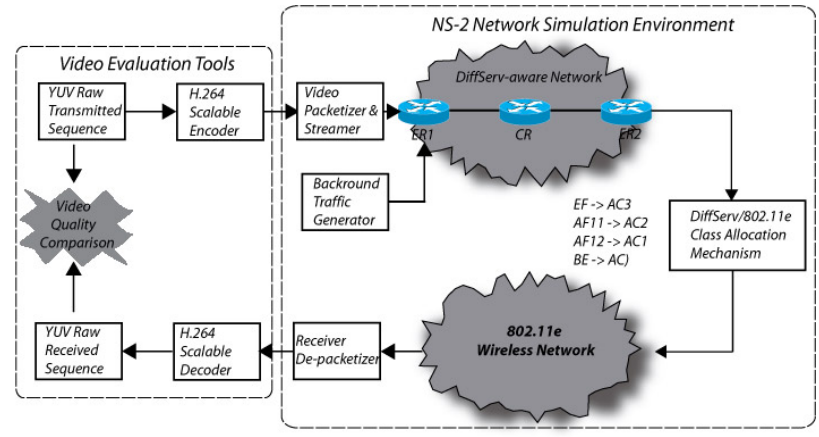

Figure 2: Simulation Setup

UDP/IP protocol stack. The $802.11 \mathrm{~b}$ is employed for the physical layer, which provides four different physical rates. In our simulation, the physical rates are fixed to $11 \mathrm{Mbps}$ for data and $2 \mathrm{Mbps}$ for control packets. Table 3 depicts the MAC Parameters for the simulations.

Additionally, the streaming node station generates background traffic (500 kbps) using constant bit rate (CBR) traffic over User Datagram Protocol (UDP). This allows us to increase the virtual collisions at the server's MAC layer. Furthermore, we include five wireless stations where each station generates $300 \mathrm{kbps}$ of data using CBR traffic in order to overload the wireless network.

A unique sequence number, the departure and arrival timestamps, and the type of payload that identify each packet. When a packet does not reach the destination, it is counted as a lost packet. Furthermore, not only the actual loss is important for the perceived video quality, but also the delay of packets/frames and the variation of the delay, usually referred to as packet/frame jitter. The packet/frame jitter can be addressed by the so called play-out buffers. These buffers have the purpose of absorbing the jitter introduced by the network delivery delays. It is obvious that a big enough play-out buffer can compensate any amount of jitter. There are many proposed techniques in order to develop efficient and optimized play-out buffer, dealing with this particular trade-off. These techniques are not within the scope of the described testbed. For our experiments the play-out buffer is set to 1000 msecs.

In order to measure the improvements in video quality by employing H.264/MPEG-4 AVC, we use the Peak Signal to Noise Ratio (PSNR) and the Structural Similarity (SSIM) [17] metrics. PSNR is one of the most widespread objective metric for quality assessment and is derived from the Mean Square Error (MSE) metric, which is one of the most commonly used objective metrics to assess the application level QoS of video transmissions [5].

Let's consider that the video sequence is represented by $v(n, x, y)$ and $v_{o r}(n, x, y)$, where $n$ is the frame index and $x$ and $y$ are the statial coordinates. The average PSNR of the decoded video sequence among frames at indices between $n_{1}$ and $n_{2}$ is given by the following equation:

$$
P N S R=10 \log _{10} \frac{V^{2}}{M S E}
$$

where $V$ denotes the maximum greyscale value of the luminance. The average $M S E$ of the decoded video sequence among frames at indices beteen $n_{1}$ and $n_{2}$ is given by: 
Table 3: 802.11 MAC Parameters

\begin{tabular}{|c|c|c|c|c|l|}
\hline Access Category & AIFS & $C W_{\min }$ & $C W_{\max }$ & Queue length & Max Retry limit \\
\hline AC3 & 50 & 7 & 15 & 50 & 8 \\
\hline AC2 & 50 & 15 & 31 & 50 & 8 \\
\hline AC1 & 50 & 31 & 1023 & 50 & 4 \\
\hline AC0 & 70 & 31 & 1023 & 50 & 4 \\
\hline
\end{tabular}

$$
M S E=\frac{1}{X Y\left(n_{2}-n_{1}+1\right)} \sum_{n=n_{1}}^{n_{2}} \sum_{x=0}^{X-1} \sum_{y=0}^{Y-1} M^{2}
$$

where $M$ is defined as:

$$
M=\left[v(x, y, n)-v_{o r}(x, y, n)\right]
$$

Note that, the PSNR and MSE are well-defined only for luminance values. As it mentioned in [5], the Human Visual System (HVS) is much more sensitive to the sharpness of the luminance component than that of the chrominance component, therefore, we consider only the luminance PSNR.

$S S I M$ is a Full Reference Objective Metric [6] for measuring the structural similarity between two image sequences exploiting the general principle that the main function of the human visual system is the extraction of structural information from the viewing field. If $v_{1}$ and $v_{2}$ are two video signals, then the $S S I M$ is defined as:

$$
\operatorname{SSIM}\left(v_{1}, v_{2}\right)=\frac{\left(2 \mu_{v_{1}} \mu_{v_{2}}+C_{1}\right)\left(2 \sigma_{v_{1} v_{2}}+C_{2}\right)}{\left(\mu_{v_{1}}^{2}+\mu_{v_{2}}^{2}+C_{1}\right)\left(\sigma_{v_{1}}^{2}+\sigma_{v_{2}}^{2}+C_{2}\right)}
$$

where $\mu_{v_{1}}, \mu_{v_{3}}, \sigma_{v_{1}}, \sigma_{v_{2}}, \sigma_{v_{1} v_{2}}$ are the mean of $v_{1}$ the mean of $v_{2}$, the variance of $v_{1}$, the variance of $v_{2}$ and the covariance of $v_{1}$ and $v_{2}$. The constants $C_{1}$ and $C_{2}$ are definde as:

$$
\begin{aligned}
& C_{1}=\left(K_{1} L\right)^{2} \\
& C_{2}=\left(K_{2} L\right)^{2}
\end{aligned}
$$

where $L$ is the dynamic range of pixel values and $K_{1}=0.01$ and $K_{2}=0.03$, respectively. [22] defines the values of $K_{1}$ and $K_{2}$.

At the first scenario, we examine the transmission of H.264 scalable video streams consisting of two layers. The BL is encoder at $256 \mathrm{Kbps}$, while the EL is encoded at $512 \mathrm{Kbps}$. As video source is used the Foreman $Y U Y$ QCIF video sequence $(176 \times 144)$ consisting of 400 frames. The underlying network for the first measurement is a simple Best Effort network, like Internet, without implementing any $Q o S$ model for guarantee end-to-end video quality. The video frame is sent every $33 \mathrm{~ms}$ for $30 \mathrm{fps}$ video. Figure 3 shows the PSNR graph for the experimental scenario described above. The Yaxis represents the $P S N R$ value in $d B$ while the Xaxis represents the frame number of video sequence.

As one may observe from Figure 3, during severe network congestion caused by interference by background traffic, the $P S N R$ values are between $10 d B$ and $12 d B$. The average value of $P S N R, P_{a v g}$ is $29.038 d B$. Note that, a frame is counted as lost also, when it arrives later than its defined playback time.

We repeat the same measurement, but instead of using a best-effort network, we use a network that implements the

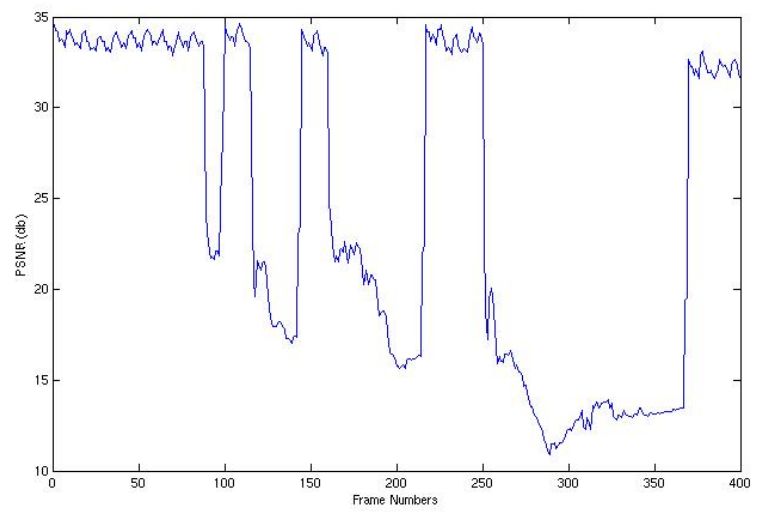

Figure 3: Scalable video transmission over besteffort networks

proposed model. The mapping of packets is based on Table 1. The DiffServ routers implement WRED queue management. In this scenario, according to Figure 4, the overall $P S N R$ is better than without using prioritization. The $P_{\text {avg }}^{-}$ value is $31.054 d B$. Figure 5 depicts the SSIM metric of both scenarios (BE and QoS-enabled networks) for foreman video sequence.

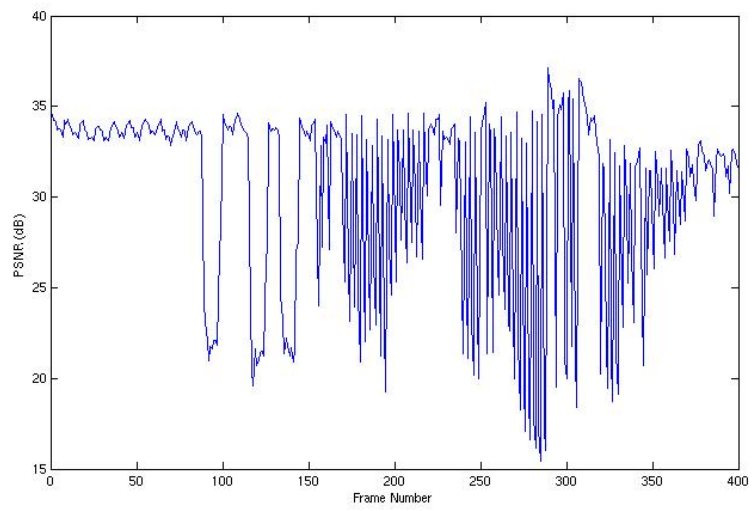

Figure 4: Scalable video transmission over DiffServ/802.11e Heterogeneous Network

We repeat the same measurement for four different $Y U V$ video sequences consisting of 300 to 2000 frames. For all the scenarios, we consider the simple but efficient error concealment scheme described in the previous section. The average $P S N R$ and SSIM for the above scenarios are shown in Table 4, where:

- in Scenario 1 we transmit scalable H.264/MPEG-4 


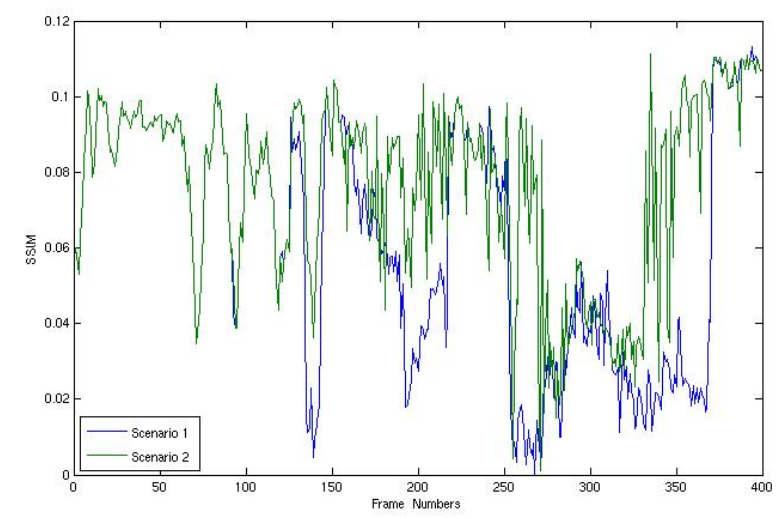

Figure 5: SSIM measurements of scalable video transmission over DiffServ/802.11e Heterogeneous Networks

Table 4: Average PSNR/SSIM for scalable H.264/MPEG-4 AVC video streams

\begin{tabular}{|c|c|c|c|c|c|}
\hline Video & \multirow{2}{*}{ Frame } & \multicolumn{2}{|c|}{ Scen.1 } & \multicolumn{2}{c|}{ Scen.2 } \\
\cline { 3 - 6 } & & $P_{\text {avg }}$ & SSIM & $P_{\text {avg }}$ & SSIM \\
\hline Highway & 2000 & 28.339 & 0.0708 & 29.762 & 0.0841 \\
\hline Mother & 961 & 28.892 & 0.0724 & 31.021 & 0.0886 \\
\hline Salesman & 444 & 28.523 & 0.0768 & 31.210 & 0.0896 \\
\hline Foreman & 400 & 29.038 & 0.0818 & 31.054 & 0.0892 \\
\hline
\end{tabular}

AVC video stream in a best effort network.

- in Scenario 2 we transmit scalable H.264/MPEG-4 AVC video stream over a DiffServ/802.11e heterogeneous network.

As it seems in Table 4, the proposed prioritization scheme improves the overall quality of the received video. By isolating the losses and the delays to packets that contain $\mathrm{B}$ and $\mathrm{C}$ partitions we can achieve significant gains to video quality. By distributing the traffic to all traffic classes, we can achieve equal or even better video quality, in the lowest price, by sending lowest traffic to the cost effective EF/AC3 traffic. From the network provider perspective, the utilization of the network is more efficient, by serving more users, at the level of quality they pay.

\section{CONCLUSIONS}

Nowadays, continuous media applications over heterogeneous all-IP networks, such as video streaming and videoconferencing, become very popular. Several approaches have been proposed in order to address the end-to-end QoS both from the network perspective, like DiffServ and 802.11e access categories, and from the application perspective, like scalable video coding and packetized prioritization mechanisms. The paper addresses the end-to-end QoS problem of scalable video streaming traffic delivery over a heterogeneous DiffServ/802.11enetwork. It proposes and validates through a number of NS2-based simulation scenarios a framework that explores the joint use of packet prioritization and scalable video coding, by evaluating scalable extension of H.264/MPEG-4 AVC, together with the appropriate mapping of 802.11 e access categories to the DiffServ traffic classes. The proposed prioritization scheme in conjuction with the proposed DiffSer/802.11e classes coupling have improvements in the overall quality of the received video, by isolating the losses and the delays to packets carrying less important partitions.

\section{REFERENCES}

[1] Network simulator v.2 (ns-2) dcf and edca extensions. http://www.tkn.tu-berlin.de/research/802.11e_ns2.

[2] Scalable extension of h.264/mpeg-4 avc. Fraunhofer Institute, Image Processing Department, available from http://ip.hhi.de.

[3] I. 802.11e. Wireless lan medium access conttrol (mac) enhancements for quality of service (qos). 802.11e draft, 2004

[4] S. Blake, D. Black, M. Carlson, E. Davies, Z. Wang, and W. Weiss. An architecture for differentiated serices. IETF RFC 2475, december 1998.

[5] A. M. R. et al. Video quality experts group: Current results and future directions. In Proc. SPIE Visual Communications and Image Processing, Perth, Australia, pages 742-753, june 2000.

[6] H. Koumaras, A. Kourtis, and D. Martakos. Evaluation od video quality based on objectively estimated metric. Journal of Communications and Networking, KICS, 7(3):235-242, september 2005.

[7] A. Ksentini, M. Naimi, and A. Gueroui. Toward an improvement of h.264 video transmission over ieee 802.11e through a cross layer architecture. IEEE Communications Magazine, pages 487-495, 2006.

[8] K. Nichols, S. Blake, F. Baker, and D. Black. Definition of the differentiated services field (ds field) in the ipv4 and ipv6 header. IETF RFC 2474, december 1998.

[9] S. Olsson, M. Stropplana, and J. Baina. Objective methods for assessement of video quality: State of the art. IEEE Transactions on Broadcasting, 43(4):487-495, 1997.

[10] S. Park, K. Kim, K. D.C., S. Choi, and S. Hong. Collaborative qos architecture between diffserv and 802.11e wireless lan. In The 57th IEEE Semiannual Vehicular Technology Conference, april 2004.

[11] T. Pliakas, G. Kormentzas, and C. Skianis. Scalable video streaming traffic delivery in ip/umts networking environments. Journal of Multimedia, 2(2):37-46, april 2007.

[12] T. Pliakas, G. Kormentzas, C. Skianis, and A. Kourtis. Mpeg- 4 fgs video streaming traffic delivery experimentation in an ip/dvb network. In $I E E E$ International Conference on Communications (ICC), june 2007.

[13] J. Reichel, H. Schwarz, and M. Wien. Joint scalable video model joint draft 6. Joint Video Team, JVT-S201, Geneva, Switzerland, april 2006.

[14] J. Reichel, H. Schwarz, and M. Wien. Joint scalable video model jsvm-6. Joint Video Team, JVT-S202, Geneva, Switzerland, april 2006.

[15] H. Schulzrinne. Rtp: A transport protocol real-time applications. RFC 1889, 1996.

[16] H. Schwarz, D. Marpe, and T. Wiegand. Overview of the scalable h.264/mpeg-4 avc extension. In IEEE 
International Conference on Image Processing, Atlanta, october 2006.

[17] Z. Wang, L. Lu, and A. Bovik. Video quality assesment based on structural distortion measurement. Signal Processing: Image Communication, special issue on Objective Video Quality Metrics, 19(2):121-132, february 2004.

[18] T. Wiegand, J. Sullivan, G. Bjontegaard, and A. Luthra. Overview of the h.264/avc video coding standard. IEEE Transactions on Circuits and Systems for Video Technology, 13(7):550-576, July 2003. 